\title{
Estado actual de ciencia de las arvenses en Nicaragua
}

\section{Current status of weed science in Nicaragua}

Freddy Alemán ${ }^{1}$, Adriana Sánchez ${ }^{2}$

${ }^{1}$ Facultad de Agronomía, Universidad Nacional Agraria, freddy@ci.una.edu.ni, ${ }^{2}$ Graduada de la carrera de Ingeniería Agrícola, Universidad Nacional Agraria

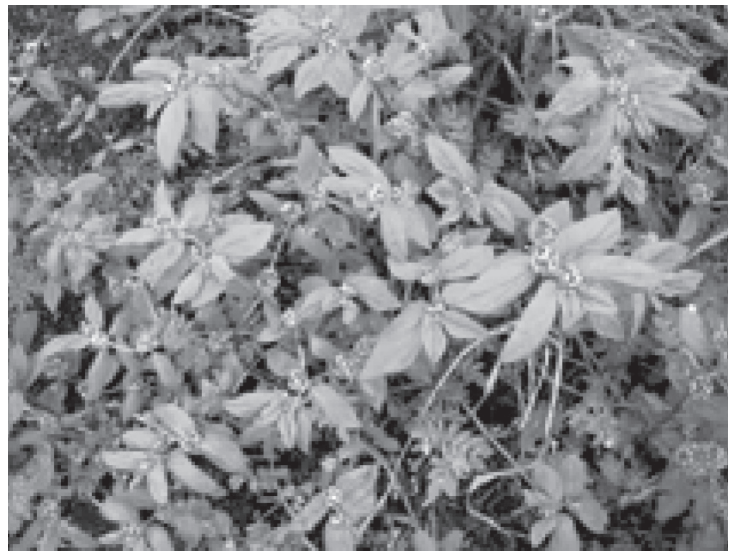

\section{RESUMEN}

En Nicaragua el estudio de las arvenses, componentes importantes de los agro ecosistema, ha sido relegado a un segundo plano. En el periodo comprendido entre agosto y septiembre del año 2010 se llevó a cabo un estudio con el objetivo de actualizar la información sobre las arvenses en Nicaragua. La investigación fue de corte transversal, la información se obtuvo a través de análisis documental y entrevistas personalizadas con actores clave de las instituciones estatales y líderes de instituciones regionales, asociaciones de productores y académicos. La mayoría de los sistemas de cultivos se ven afectados por la presencia de arvenses y existe predominio de prácticas de manejo convencional que propician mayor infestación de arvenses. Trece especies de malezas son consideradas altamente problemáticas en la agricultura nicaragüense, y trece muy problemáticas. La práctica de manejo de arvenses utilizada por pequeños agricultores es manual, a través de la utilización de implementos livianos y arranque a mano. Agricultores medianos y grandes utilizan herbicidas como paraquat, glifosato y 2,4 $\mathrm{D}$, algunos de ellos de uso restringido en muchos países. En los últimos diez años ha habido un incremento en el uso de herbicidas, y hay sistemas de cultivo como arroz, sorgo, caña de azúcar, café y cultivos oleaginosos (soja, maní y ajonjolí) que dependen de los herbicidas para reducir las arvenses. Son pocos los profesionales que trabajan en ciencia de las malezas, así como los programas y proyectos de investigación en curso que consideran la temática. No existe información sobre arvenses acuáticas, la práctica de control biológico es inexistente, y pocos programas de certificación de semillas cumplen con las normas establecidas que incluyen un especial manejo de arvenses. A nivel nacional existen documentos de reglamentación, así como convenios o tratados internacionales que influyen en las opciones de manejo de las arvenses.

Palabras clave: malas hierbas, control de malezas, medidas fitosanitarias, normas técnicas, protección de plantas

Recibido: 20 de noviembre 2014

Aceptado: 13 de julio 2015

\section{ABSTRACT}

In Nicaragua, the study of the weeds, important components of the agro ecosystem, has been neglected to a second place. In the period comprised between August and September 2010, a study was conducted aimed to update the information on weed science in Nicaragua. The research was transversal; the information was obtained through document analysis and interviews with key stakeholders from state institutions, leaders of regional institutions, producer associations and academia. The results show that most of the farming systems in Nicaragua are affected by weeds presence, in which there is predominance of conventional management practices that promote greater weeds infestation. Thirteen weed species are considered highly problematic in Nicaraguan agriculture, and thirteen as very problematic. The weed management practice used by small farmers is manual control, with the use of light tools and manual pulling. Medium and large farmers use herbicides which include paraquat, glyphosate and 2,4-D, some of them restricted in many countries. In the last ten years there has been an increase in the use of herbicides, existing farming systems such as rice, sorghum, sugar cane, coffee and oil crops (soybean, peanut and sesame) that depend on them to control weeds. According to the informants, few professionals are working in weed science, as well as there are few programs and ongoing research projects that consider the subject. Information on aquatic weeds and biological control practice are nonexistent, and few seed certification programs fulfill established standards of a particular weed management to not affect the seed quality. Nationally there are regulatory or policy documents on weeds, as well as international agreements or treaties subscribed by the country, which influence the management options of these plants. 


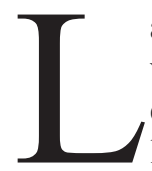

a importancia de un adecuado manejo de la vegetación adventicia en la producción agrícola está fuertemente sustentada. El manejo eficiente de las plantas que colonizan los campos cultivados es imperativo para lograr una producción rentable y de calidad. Diferentes métodos de control se ha utilizado para el manejo de la arvenses, sin embargo, en la agricultura nicaragüense, el manejo de estas plantas es todavía una de las principales limitantes para la producción de cultivos.

Muchos autores coinciden en plantear la importancia de las arvenses como componentes importantes de los agroecosistemas agrícolas. Como individuos propios del sistema, cumplen un papel importante en el balance del agroecosistema, e.g., son importantes en el reciclaje de nutrientes, en la preservación de la diversidad biológica, en el control de la erosión y en la conservación de la humedad (Altieri, 1983, Hart, 1985). Estas características importantes de las arvenses como reguladores biológicos obliga a su estudio, a conocer más sobre la función bilógica que desempeñan en los agroecosistemas agrícolas, y a entender aspectos bioecológicos de su funcionamiento, con el propósito de evitar la interferencia que pueden ocasionar a las plantas cultivadas, y a la vez, obtener beneficios de la presencia de las mismas en los campos cultivados.

A pesar de la importancia de las arvenses en el agro ecosistema, y de jugar un papel primordial en el balance del mismo, esta disciplina ha sido descuidada por el sistema productivo nicaragüense. Los productores agropecuarios orientan su control a la erradicación de los campos, sin considerar posibles ventajas que pueden brindar al sistema. Por otro lado, los programas de investigación no las consideran dentro de sus actividades, limitando la posibilidad de desarrollar prácticas efectivas para reducirlas de los campos, sin deterioro del ambiente.

Ante esta problemática se hace necesario conocer el estado actual de ciencia de las arvenses en los campos cultivados en Nicaragua, conocer que se está haciendo para darles la importancia que se merecen como componentes integrales de los agroecosistemas agrícolas, los esfuerzos desarrollados desde la academia, desde los sectores productivos y desde el gobierno central para promover que puedan desplegar su papel en beneficio del sistema en general y de la producción de los cultivos.

El objetivo del presente estudio fue actualizar la información sobre manejo de arvenses en Nicaragua, problemas actuales y potenciales y prácticas de manejo utilizadas por los agricultores, conocer los esfuerzos desarrollados por el sector académico para mantener o mejorar las temáticas en el currículo universitario, esfuerzos desarrollados por el estado nicaragüense para reducir la presencia de las mismas en los campos cultivados, actualizar la información sobre profesionales que trabajan en ciencia de las arvenses y conocer acerca de aspectos regulatorios o de políticas que influencien el manejo de las mismas.

\section{MATERIALES Y MÉTODOS}

El trabajo de investigación fue establecido entre los meses de agosto a septiembre del año 2010. El estudio fue de corte transversal, orientado a analizar la situación actual de ciencia de las arvenses en Nicaragua.

La información se obtuvo a través de revisión documental, para lo cual se revisaron informes de instituciones estatales y académicas. Además, se realizaron entrevistas personalizadas con actores claves de las instituciones estatales y líderes de instituciones regionales en Nicaragua. También se entrevistaron líderes de asociaciones de productores y académicos encargados de la cátedra de arvenses de diferentes Universidades del país.

Inicialmente hubo una selección de informantes clave, que incluían a personas de universidades, organismos internacionales, instituciones gubernamentales, etc. Esta lista inicial no se cumplió totalmente, ya que era difícil ponerse en contacto con algunos representantes de algunas instituciones. Sin embargo, fueron sustituidos por personal calificado con conocimientos de manejo de arvenses en Nicaragua. Las partes interesadas incluyen a profesionales de la academia (3), gobierno (3), asociaciones de agricultores (2), organismos internacionales (2), ONG (2) y consultor independiente (1).

Al inicio de la investigación hubo representantes de organismos internacionales a quienes se tuvo la intensión de entrevistar, sin embargo, estas personas estaban más involucradas en aspectos administrativos, por lo que se les pidió designar a otra persona para la entrevista, un informante específico que se sabía podría brindar información acorde a lo esperado.

La información brindada por los informantes clave fue grabada, para luego ser trascrita y analizada en sesiones de gabinete.

\section{RESULTADOS Y DISCUSIÓN}

Infestación y manejo de arvenses en los sistemas de cultivo Sistemas de cultivo más afectados por las arvenses. Los agricultores de pequeña escala tienen dificultades para el manejo de las arvenses. La mayor parte del tiempo que dedican a la producción agrícola se orienta a reducir las malas hierbas del campo, con lo cual impiden la utilización de su tiempo en otras actividades propias del cultivo. Los métodos utilizados por estos agricultores para controlar las arvenses son mecánicos, para lo cual utilizan azadón y machete. La práctica se lleva a cabo en suelos con buena humedad y fertilidad, lo que suele aumentar la densidad de las especies perennes como coyolillo (Cyperus rotundus L), y pasto bermuda (Cynodon dactylon (L.) Pers). La práctica mecánica de cortar las estructuras vegetativas rompe la dominancia apical, lo que activa las estructuras vegetativas de las arvenses perennes, ocasionando mayor difusión de estas especies (Dinarte et al., 1993). 
Otros sistemas de cultivo muy afectados por las arvenses son los sistemas de mediana y gran producción. La producción de arroz bajo riego, los cultivos de sorgo y oleaginosas, así como la producción de hortalizas a pequeña escala (cebolla, tomate, zanahoria, etc.) y la producción de frijol común.

Prácticas de laboreo para el manejo de las arvenses. Una de las prácticas más comunes enfocadas al manejo de las arvenses en Nicaragua es el laboreo del suelo. De acuerdo a la percepción de los entrevistados esta práctica, lejos de ser beneficiosa, en la mayoría de los casos propicia una mayor infestación de arvenses. El uso del arado y rastra (labranza convencional) permite una infestación de arvenses con alta calidad y cantidad. Otro factor que promueve la infestación de arvenses es la combinación de laboreo convencional y el uso de herbicidas, e.g., en la producción de arroz, el uso de diferentes herramientas mecánicas para la perturbación del suelo y el uso y abuso de herbicidas conduce a infestaciones de arvenses, en muchos casos difíciles de manejar (Dinarte et al., 1993).

El uso de labranza convencional con arado y rastra para el manejo del suelo favorece el crecimiento de plantas nocivas $\mathrm{y}$ el desarrollo de nuevas especies. La labranza excesiva no sólo permite mayor difusión de las estructuras vegetativas de las arvenses perennes, pero también representa mayor probabilidad de germinación de las semillas (Staver, 1991).

Algunas evidencias de investigación muestran que la práctica de siembra directa tiene un impacto sobre las poblaciones de arvenses. Esta práctica propicia cambios en la flora de arvenses, reduce el número de individuos por especie, el número de especies, hay predominio de gramíneas, y a mediano plazo trae serios problemas con arvenses bienales y perennes, que aparecen después de un período de tres a cuatro años (Alemán, 2001). en Nicaragua

Arvenses comunes en los sistemas de cultivo en Nicaragua. La información muestra que existe un grupo particular de arvenses que se encuentran asociadas a los cultivos en Nicaragua. Las especies mencionadas se repiten entre los entrevistados, y únicamente para el cultivo de arroz se refieren a una flora particular.

Los problemas de arvenses específicas difíciles de controlar están asociados a diversos factores, tales como cultivos y su manejo, y las características biológicas y ecológicas de las especies. En ocasiones el problema se centra en una especie específica, como coyolillo, en la producción de maíz; o pasto Johnson (Sorghum halepense) en la producción de sorgo. Sin embargo, en muchos casos el problema es ocasionado por la asociación de especies que crecen junto al cultivo. Es el caso de las especies de hoja ancha comunes en la horticultura y en la producción de frijol común, o especies de hoja fina que afectan la producción de arroz y maíz.

En el cuadro 1, se muestra una lista de las arvenses, que según los informantes, son más comunes en los sistemas de cultivo en Nicaragua, se indica además, el nombre común y el cultivo en el que comúnmente se le encuentra.

Cuadro 1. Especie, familia, nombre común y cultivo afectado por arvenses comunes en sistemas de cultivo

\begin{tabular}{|c|c|c|c|}
\hline Especie & Familia & Nombre común & Cultivo afectado \\
\hline Euphorbia hetherophilla L. & Euphorbiaceae & pastorcillo & Frijol común, Soya y Maíz \\
\hline Cyperus rotundus $\mathrm{L}$. & Cyperaceae & coyolillo & Frijol común, Maíz y Caña de azúcar \\
\hline Melampodium divaricatum D.C. & Asteraceae & flor amarilla & Frijol común, \\
\hline Ixophorus unicetus Schlecht & Poaceae & zacate dulce & caña de azúcar, Maíz y Sorgo \\
\hline Ipomoea tilicaceae (Wild) Choisy & Convolvulaceae & batatilla & Café y Sorgo \\
\hline Echinochloa colonum (L.) Link. & Poaceae & grama de agua & Arroz \\
\hline Sagitaria trifolia Engels & Alismataceae & Saetilla & Arroz \\
\hline Ischaemun rugosum Salisb. & Poaceae & falsa caminadora & Arroz \\
\hline Alternantera sessilis & Amaranthaceae & camarón & Café \\
\hline Commelina diffusa Burm. F. & Commelinaceae & siempre viva & Café y Arroz \\
\hline Galinsoga $\mathrm{sp}$ & Asteraceae & cominillo & Café \\
\hline Physalis angulata $\mathrm{L}$. & Solanaceae & popa & Tomate \\
\hline Chloris clorideae & Poaceae & zacatón & Caña de azúcar \\
\hline Sida acuta & Malvaceae & escoba lisa & Cultivos anuales \\
\hline Blatimora recta & Asteraceae & me caso, no me caso & Cultivos anuales \\
\hline Cyperus iria & Cyperaceae & navajuela & Arroz de riego \\
\hline Eichornia crassipes & Pontederiaceae & Jacinto de agua & Mantos acuáticos \\
\hline Rotboellia conchinchinensis & Poaceae & caminadora & Maíz, Caña de Azúcar, Arroz \\
\hline Pistia stratiotes & Araceae & lechuga de agua & Mantos acuáticos \\
\hline Digitaria sanguinalis & Poaceae & paja peluda & Cultivos anuales \\
\hline Oryza sativa & Poaceae & arroz maleza & Arroz de riego \\
\hline Sorghum halepense & Poaceae & invasor & Maíz, Sorgo \\
\hline
\end{tabular}

Arvenses de más difícil control en Nicaragua Sistemas de cultivo mayormente afectados por las arvenses. Los entrevistados coinciden en que si no se ejecutan prácticas de manejo de arvenses, las mismas rápidamente colonizan la mayoría de los cultivos. Hay algunas especies de arvenses con las que los agricultores deben tener cuidado, debido a la característica de ser arvenses invasoras. Muchos cultivos en la zona del pacífico son altamente infestados por coyolillo o 
por batatilla (Ipomoea nil), que es una maleza invasora que afecta pastizales, cultivos oleaginosos y cultivos perennes. La escoba lisa (Sida acuta) es una maleza invasora en cultivos de hoja ancha anuales, como frijol y maní. La caminadora (Rottboellia cochinchinensis) es una maleza invasora en caña de azúcar; y pasto Johnson en sistemas de producción de sorgo.

Prácticas de manejo de arvenses utilizadas por los agricultores. El manejo de arvenses en Nicaragua es deficiente. Las prácticas utilizadas por los pequeños agricultores datan desde el siglo pasado. Los informantes coinciden en expresar que el uso de control mecánico utilizando machete, azadón, y arranque manual es la principal vía utilizada para el control de arvenses en Nicaragua. La actividad de control de arvenses involucra gran parte del tiempo que el agricultor dedica al manejo de la producción agrícola. Por otro lado, los medianos y grandes agricultores utilizan herbicidas para controlar las arvenses. Los medianos agricultores utilizan generalmente herbicidas post-emergentes, no selectivos, los cuales en la mayoría de los casos tienen uso restringido en países en desarrollo, i.e., paraquat. Agricultores de avanzada utilizados herbicidas selectivos de pre-emergencia, en cultivos como caña de azúcar, arroz, oleaginosas, sorgo, etc.

Limitaciones propias del manejo de arvenses. La actividad de control de arvenses en Nicaragua tiene diversas restricciones. En Nicaragua hay pocas instituciones que destinan financiamiento a la producción agrícola, lo cual no permite desarrollar prácticas avanzadas para el manejo der las arvenses.

Sin embargo, a juicio de los entrevistados, el principal factor que afecta el control de arvenses en Nicaragua es la falta de conocimiento y tecnología en el manejo de las mismas. Hay pocas instituciones que trabajan en ciencia de las arvenses, y en generar y difundir conocimientos en esta área. Esa es la razón que ocasiona que a la fecha se implemente un pobre manejo de arvenses basado en la utilización de prácticas tradicionales.

Herbicidas más utilizados por los agricultores. En Nicaragua, los herbicidas más utilizados por los agricultores son totales no selectivos como paraquat y glifosato; y hormonales como 2,4 D. En la producción de frijol común se utilizan algunos herbicidas para el control de arvenses de hoja fina, e.g., Fluazifop butilo, y herbicidas para controlar arvenses de hoja ancha, como Fomesafen. La producción de arroz ha introducido algunos herbicidas del grupo de las sulfonilureas, como metil-Metsulfurón y del grupo ariloxifenoxi propionatos, como fenoxaprop-P-etil. Herbicidas como el propanil (en arroz), ametrina (en caña de azúcar), atrazina (en caña de azúcar, maíz, sorgo y piña) fueron importantes en el pasado para el control de arvenses en Nicaragua.
Muchos sistemas agrícolas utilizan herbicidas no selectivos como el paraquat y el glifosato. Agricultores involucrados en la producción de sorgo hacen uso de herbicidas selectivo de pre-emergencia como metolacloro y pendimetalin. Por otro lado, los agricultores involucrados en la producción de arroz utilizan herbicidas para el control de arvenses de hoja fina (poáceas y ciperáceas). Estos herbicidas pertenecen en su mayoría a la familia de las sulfonilureas.

El uso de herbicidas ha cambiado en los últimos diez años. Sistemas de producción que dependen de herbicidas continúan usando el mismo producto (herbicidas no selectivos). Por otro lado agricultores de avanzada dependen de la utilización de herbicidas. Esto ha promovido y aumentado el uso de herbicidas, comparado con la utilización existente hace diez años.

El alto costo de la mano de obra y la eficiencia de control a través de la utilización de herbicidas han permitido que productores de avanzada dependan del uso de herbicidas. No es el caso de los pequeños agricultores para los cuales no es rentable el uso de herbicidas.

Sistemas de cultivo basados en el uso de herbicidas para controlar las arvenses. Medianos y grandes productores que cultivan arroz, sorgo, caña de azúcar, café y cultivos oleaginosos (soja, maní y ajonjolí) dependen del uso de herbicidas. Toda la información acerca del uso, problemas asociados, toxicidad, control de arvenses, etc., de los herbicidas ha sido desarrollada en el extranjero. No hay instituciones que hayan estudiado aspectos relativos a los herbicidas que se introducen a Nicaragua. En vista de lo anterior es necesario llevar a cabo investigaciones orientadas a determinar los aspectos mencionadas.

Problemas regionales en el manejo de las arvenses. Uno de los temas importantes de la región en materia de arvenses es la producción de semillas de cultivos. La producción de semilla requiere un especial manejo con el fin de garantizar la calidad de la misma. En este sistema de producción, las arvenses se constituyen en un problema especial, debido a que la competencia de estos individuos, además de reducir la calidad, contamina el producto, con semillas y partes vegetativas de las arvenses.

Otro aspecto importante desde el punto de vista regional es la introducción de plantas exóticas de un país a otro. Los ministerios y oficinas de cuarentena en la región deben estar conscientes de los posibles problemas que podrían surgir si se introducen especies de arvenses. Avena silvestre (Avena fatua), y la commelina (Commelina bengalensis) son dos especies que se reportan en los países vecinos, por tanto se requiere establecer medidas y prácticas de prevención para evitar su introducción.

Otro problema potencial es la interferencia de arvenses. Se tiene que estudiar la asociación de arvenses que interfieren con el normal crecimiento de los cultivos y 
sistemas de cultivos en Nicaragua, así como, la población de arvenses que afectan al establecimiento normal de los cultivos. También, se debe estudiar la dinámica de las arvenses y las ventajas que proporcionan a los sistemas de cultivo y al agro ecosistema en general.

No se sabe mucho acerca de características bioecológicas de la asociación de arvenses. Se necesita saber más acerca de la dinámica de poblaciones de arvenses, banco de semillas de arvenses, y los períodos y umbrales de control de las arvenses.

Materiales técnicos divulgativos sobre problemas de arvenses y prácticas de manejo. En Nicaragua no existen folletos técnicos sobre los problemas de arvenses y acerca de las prácticas agrícolas para el manejo de las mismas. Se cuenta con algunos boletines técnicos que tratan sobre el manejo de los diferentes cultivos (sorgo, arroz, frijol, maíz, etc.), con una sesión de prácticas de manejo de arvenses. Años atrás se contaba con materiales divulgativos que explicaban cómo manejar las arvenses en diversos cultivos, sin embargo, esta información no se ha actualizado.

Científicos que trabajan en ciencia de las arvenses en Nicaragua. Los informantes proveyeron información sobre profesionales que trabajan en ciencia de las Arvenses. La mayoría de los profesionales mencionados desempeñaron funciones de investigación y trasferencia de conocimientos en ciencia de las arvenses, sin embargo, en la actualidad desempeñan otras funciones relacionadas al agro nicaragüense.

Hace quince años, en Nicaragua, se produjo un movimiento de especialistas en arvenses. Muchos herbólogos trabajaban en las diferentes áreas de la ciencia de arvenses. Algunos trabajaban en la industria, otros en el manejo agroecológico de arvenses y otros en el manejo integrado de arvenses (MIA). En ese entonces se creó la asociación de malezólogos de Nicaragua, sin embargo, solo funcionó durante dos años. Hoy en día muchos de los que trabajaban con arvenses se dedican a otras actividades del agro. En este momento, en Nicaragua, la ciencia de las arvenses está totalmente descuidada, y hay poco interés en su estudio.

Actividades o proyectos de investigación en curso sobre manejo de arvenses en Nicaragua. La Universidad Nacional Agraria cuenta con programas y proyectos de investigación que consideran actividades de manejo y control de arvenses, principalmente aspectos de manejo cultural de las mismas. Muchos de estos estudios están orientados a la graduación de estudiantes, por lo que son importantes desde el punto de vista académico, pero carecen de respuesta para problemas sentidos por la producción agrícola de Nicaragua.

El Centro Agronómico de Investigación y Enseñanza (CATIE), en asociación con la Universidad Nacional Agraria ha desarrollado programas de investigación, en los que las arvenses son aspectos importantes dentro de los estudios. Estas investigaciones consideran el manejo cultural de arvenses como una forma de reducir la infestación de las mismas de las áreas cafetaleras.

La Asociación Nacional de Productores de Arroz (ANAR), está desarrollando investigaciones sobre manejo de arvenses en las plantaciones de arroz, con resultados satisfactorios, sin embargo, lo resultados de dichas investigaciones son poco visibles.

Como país, Nicaragua no cuenta con una estrategia para hacer frente a los problemas que ocasionan las arvenses en los campos cultivados. En la protección de plantas, se han priorizado los estudios sobre las plagas y enfermedades, dejando a un segundo plano el estudio de las arvenses. Sin embargo, en la práctica los problemas de arvenses son de interés primordial.

Hay pocos programas de investigación que estudien las arvenses de manera integral, en los cuales se les considere como componentes importantes de los ecosistemas agrícolas. Existen pocos estudios de investigación, divulgación y extensión orientados a proporcionar la verdadera dimensión que estas plantas tienen en los campos cultivados, no existen trabajos colaborativos entre las instituciones del sector agropecuario que permitan aumentar el impacto de estos estudios; y no se vislumbra la información que permita conocer más acerca de la biología y ecología de las arvenses.

Uso de control biológico en el manejo de las arvenses. El control biológico de arvenses no se ha implementado en Nicaragua. La pregunta para los informantes clave estaba referida al control biológico clásico de arvenses, que es la introducción de organismos exóticos de un país donde se originan. En Nicaragua no hay experiencia con control biológico clásico, ni con control biológico aumentativo (producción a nivel de laboratorio de un organismo que se ha determinado afecta a determinada planta nativa). La anterior ha sido una práctica común para el control de insectos, pero no para las arvenses.

En Nicaragua se ha experimentado con control biológico natural para reducir las arvenses de los campos cultivados. Se han utilizado cultivos de cobertura para reducir la infestación de arvenses (Aguilar, 2000), residuos de plantas que limitan la infestación de arvenses, plantas de cultivo que se defienden eficientemente de estas especies, $\mathrm{y}$ arreglos de plantas que permiten un cierre temprano del entrecalle reduciendo el establecimiento de las arvenses.

Los entrevistados que respondieron a la pregunta, la analizaron, en su mayoría, orientada al control de las arvenses a través de control biológico natural.

Problemas de infestación y manejo de arvenses relacionados a la producción de semilla. Este tema es relevante en la agricultura nicaragüense, en los sistemas de cultivo dedicadas a la producción de semillas, los agricultores deben 
tener especial manejo de los cultivos con el fin de reducir la competencia de las arvenses que puede reducir la calidad de la semilla. En la producción convencional, los agricultores tienen la oportunidad de utilizar el período crítico de control de arvenses para establecer prácticas de control, sin embargo, en áreas de producción de semillas, el cultivo debe permanecer limpio durante todo el ciclo, o permitir presencia de arvenses hasta ciertos niveles. Otro problema que los agricultores deben enfrentar es la contaminación de la semilla de cultivo con semillas y estructuras vegetativas de arvenses. Es común en la producción de semillas de arroz que muchos de las arvenses pasen directamente junto con las semillas de arroz a las áreas de siembra. También es posible que las estructuras vegetativas contaminen las semillas de cultivos, las cuales germinan una vez que el cultivo es plantado.

El primer principio para controlar las arvenses es el uso de semilla limpia. En general, la forma más común y eficaz para la introducción de arvenses es a través de la venta y distribución de semillas comerciales mezclado con semillas de arvenses. El conocimiento de este aspecto, ha propiciado el establecimiento de normas jurídicas destinadas a evitar la introducción y propagación de arvenses, las cuales se han implementado de forma gradual en Nicaragua. Un problema a resolver es el compromiso de las empresas de distribución de semillas para asegurar la calidad de los materiales.

Algunas especies de arvenses que potencialmente están relacionadas a la producción de semilla son las siguientes, falsa caminadora (Ischaemun rugosum), se mezcla con las semillas de arroz y pasa directamente durante el proceso de cosecha y beneficiado; pasto Johnson (Sorghum halepense), es comúnmente mezclada con semillas de sorgo durante el proceso de cosecha. En pastizales, es fácil que las semillas de pasto estén contaminadas con muchas arvenses silvestres. Esto ocurre a lo interno, pero también con semillas que vienen del extranjero. Esta es la razón por la cual la oficina de vigilancia fitosanitaria posee normas y procedimientos para evitar la introducción de especies exóticas en semillas importadas del extranjero. Semillas de Brachyaria spp es comúnmente contaminado con plantas silvestres como pasto retana (Ischaemun ciliaris) o zacate jaragua (Hiparhenia ruffa).

\section{Arvenses acuáticas}

Arvenses acuáticas consideradas posibles amenazas. En los sistemas de cultivo, los problemas de arvenses acuáticas se circunscribe a la producción de arroz. Muchas plantas acuáticas crecen junto al cultivo, entre ellas, grama de agua (Echynocloa colona), Saetilla (Sagitaria sp), y azul (Hetheranthera sp). También hay problemas potenciales en áreas no agrícolas con especies tales como. Jacinto de agua (Eichornia cassipes) y lechuga de agua (Pistia stratiotes), que son las plantas acuáticas más comunes que afectan áreas con cuerpos de agua como lagos, estanques, depósitos de agua, etc.
Prácticas de manejo contra arvenses acuáticas. En los sistemas de cultivo, la práctica más común es la preparación del suelo a través del fangueo. El uso de diferentes implementos operados en diferentes direcciones destruyen las estructuras vegetativas de las plantas acuáticas las que al final sufren necrosis en sus tejidos. La preparación adecuada del suelo ejerce algún tipo de control de arvenses acuáticas mediante la creación de un entorno favorable para la emergencia del cultivo. Tal práctica es eficaz cuando los campos tienen infestación de baja a media de hierbas agresivas tales como arroz rojo (Oryza sativa). Esta práctica implica el anegamiento prolongado el cual tiene buena eficacia en la reducción de las especies de arvenses difíciles de manejar.

En cuerpos de agua como reservorios de agua, lagos, etc., la única manera de reducir la infestación y la difusión de arvenses acuáticas es a través de la extracción directa mediante extracción física.

\section{Aspectos regulatorios}

Documentos nacionales - regionales de reglamentación o de política sobre arvenses. El gobierno de Nicaragua ha definido leyes que ejercen regulación sobre aspectos relacionados a la introducción de arvenses exóticas. Lo mismo es válido para normas y reglamentos que se aplican en la producción de semillas, las cuales establecen los niveles de tolerancia para la presencia de arvenses en áreas de producción de semilla certificada.

Nicaragua cuenta con leyes que regulan la importación y venta de semillas comerciales, laboratorios para el estudio de muestras de semillas y un sistema organizado para la inspección de semillas, así como tratados internacionales relacionados con la conservación del medio ambiente.

Algunas de las normas y reglamentos son: norma o reglamento técnico para la producción y comercialización de semilla certificada de granos básicos (arroz, sorgo, frijol y maíz común) y soja; ley Número 274 y su reglamentación, para la regulación y control de plaguicidas, sustancias tóxicas, peligrosas y otras similares, donde se incluyen los herbicidas; norma técnica para la regulación de la práctica de quema en caña de azúcar, NTON 05 030-06. La norma Técnica Nicaragüense $17002-02$, la cual establece los procedimientos para la toma de muestras y preparación de productos derivados de la planta, la cual en algunas de sus cláusulas se refiere las arvenses. También se cuenta con la norma Técnica Obligatoria Nicaragüense norma 11 00100 para la certificación fitosanitaria, que tiene por objeto establecer las disposiciones, requisitos y procedimientos que regirán la actividad de certificación fitosanitaria para la exportación de productos agrícolas frescos y procesados. 
Instituciones involucradas en el ciencia de las arvenses a nivel nacional. Entre las instituciones involucradas en ciencia de las arvenses en Nicaragua, destacan: MAG (Ministerio de Agricultura), INTA (Instituto Nacional de Tecnología Agropecuaria), Universidad Nacional Agraria (UNA), Asociación de Agricultores (UNAG, UPANIC ANAR, AMPROSOR, etc.), y organizaciones regionales de cooperación (CIAT, CATIE, IICA, FAO, etc.).

Convenios - tratados internacionales suscritos por el país, que pueden influir en las opciones de manejo de arvenses. Nicaragua como país ha firmado tratados internacionales en materia de comercio. Nicaragua se ha unido a otros países en el mundo y ha firmado acuerdos de libre comercio con los países de la Unión Europea, un tratado de libre comercio con América del Norte, el MERCOSUR: Mercado Común del Sur y: Mercado Común Centroamericano. Estos acuerdos comerciales incluyen diferentes aspectos tendientes a minimizar la introducción de organismos nocivos para las actividades agrícolas, que se aplica también al caso de las arvenses.

\section{CONCLUSIONES}

A pesar de ser componentes importantes del agro ecosistema y constituirse en potenciales problemas para la producción de los cultivos, en Nicaragua, las arvenses son poco estudiadas. En la agricultura nicaragüense predominan prácticas tradicionales de control basadas principalmente en el uso de implementos mecánicos y el control manual. Además, la ciencia de las arvenses se constituye en una de las áreas con menor formación profesional de parte de los profesionales del agro nicaragüense, careciendo de programas de especialización y programas de investigación enfocados a generar conocimiento en el área.

Es necesario capacitar a técnicos y productores sobre el manejo de arvenses en los campos agrícolas. Además, hay que fortalecer el currículo universitaria en aspectos de ciencia de las arvenses, pero también educar a la sociedad sobre la importancia de un eficiente manejo de estas plantas.

Realizar estudios sobre manejo integrado de arvenses y analizar y comprender el papel que juegan las arvenses en los sistemas agrícolas.

Actualmente se ha descuidado la práctica de manejo de estas especies. Hay pocos programas de investigación que estudian las arvenses de manera integral, y que las consideren como componentes importantes de los ecosistemas agrícolas. Hay pocos estudios de investigación, poca divulgación de lo que se está haciendo y poca colaboración entre las instituciones para mejorar el impacto de estos estudios.

\section{AGRADECIMIENTO}

Se agradece el soporte financiero de FAO para la realización de este estudio. Extensivo agradecimiento para los profesionales y técnicos que brindaron información para derivar las conclusiones del estudio.

\section{REFERENCIAS BIBLIOGRÁFICAS}

Alemán Z, F. 2000. Studies on bean—maize production systems in Nicaragua. Doctoral thesis. AGRARIA. 239. ISSN 1401-6249, ISBN 91-576-5788-2

Aleman, F. 2001. Common bean response to tillage intensity and weed control strategies. Agronomy Journal 93: 1-7.

Altieri, MA.1983. Agroecology. The scientific basis of alternative agriculture. Berkerley, California. 162p.

Aguilar, V. 2001. Selective weed and ground cover management in a coffee plantation with shade trees in Nicaragua. Doctoral thesis. AGRARIA. 269. ISSN 1401-6249, ISBN 91-576-5799-8

Dinarte, S; Staver, Ch; Aker,Ch; Sarria, M; Martinez, R. 1993. Labranza en seco para el control de Cyperus rotundus L. en campos de agricultores. Nota Técnica. Agronomía Mesoamericana 4: 53-57.

Hart, R. 1985. El subsistema malezas, en Agro-ecosistemas, Conceptos Básicos. CATIE. Turrialba, Costa Rica. P. 103 - 110.

Staver, Ch. 1991. La labranza en seco para el control de Cyperus rotundus en el occidente de Nicaragua: el efecto de método de labranza y tiempo de exposición. CATIE, Turrialba (Costa Rica). Programa I: Mejoramiento de Cultivos Tropicales. 